Techniques \& Culture

\title{
La planche à voile
}

\section{Philippe Lacombe}

\section{OpenEdition}

Journals

Édition électronique

URL : https://journals.openedition.org/tc/154

DOI : $10.4000 /$ tc. 154

ISSN : 1952-420X

\section{Éditeur}

Éditions de l'EHESS

\section{Édition imprimée}

Date de publication : 1 juin 2002

ISSN : 0248-6016

\section{Référence électronique}

Philippe Lacombe, «La planche à voile », Techniques \& Culture [En ligne], 39 | 2002, mis en ligne le 29 avril 2005, consulté le 29 septembre 2022. URL : http://journals.openedition.org/tc/154 ; DOI : https:// doi.org/10.4000/tc. 154

Ce document a été généré automatiquement le 29 septembre 2022

Tous droits réservés 


\title{
La planche à voile
}

\author{
Philippe Lacombe
}

«Si l'histoire des pratiques de jeux et de sports
s'est récemment renforcée, c'est surtout par
l'investigation des transformations de leurs
usages sociaux. Très peu de recherches par
contre sur le développement des innovations
techniques, sur leurs chronologies, leurs
contextes. Cette histoire reste à faire. "

(Vigarello 1988).

1 Est-ce en raison de sa jeunesse ?, toujours est-il que l'histoire de la planche à voile n'a jusqu'à présent guère bénéficié de contributions savantes. Si la succession des innovations en matière de flotteurs, gréements, accessoires ou vêtements paraît simple, une reconstitution approximative risque d'être partielle, ou d'occulter quantité de tentatives et de balbutiements non retenus dans la genèse chaotique de l'engin. Nous essaierons d'en pointer quelques développements au passage.

2 En effet, nous retiendrons davantage la perspective synchronique en laquelle se recoupent différents champs de la vie sociale et qui en interroge les acteurs. Les usages de la motricité humaine et du corps s'inscrivent de toute évidence dans les conditions sociales, techniques et culturelles.

3 Les engins sportifs relèvent de l'analyse du changement technique comme d'autres objets ordinaires, ludiques ou professionnels. Aujourd'hui, différents cadres permettent de restituer l'histoire des objets techniques : bénéficiant pour partie d'une autonomie relative, cette histoire est nécessairement pluridisciplinaire car en interaction avec l'économie, la sociologie, la politique... L'objet peut, et cela témoigne d'engagements théoriques distincts, être considéré comme «individu dans le monde des objets [...] produit d'une organisation sociale [...] objet de consommation » ou encore "élément utilitaire dans le système d'utilisation » (Rosmorduc 1997).

4 L'invention de la planche à voile est, au même titre que celle de la bicyclette à la fin du XIXe siècle, une révolution sociale, technique et culturelle. L'homologie technique est saisissante : dans les deux cas, sont absentes des expé-riences transférables ou proches. 
Aucun engin préexistant ne permettait d'aller ainsi sur l'eau, seul, de s'y déplacer debout et rapidement; Joël de Rosnay y voit une filiation, celle de la glisse, du surf. Pourtant la planche capitalise essentiellement l'expérience du dériveur, celle de la verticalité vélique. Certains auteurs considèrent que l'engin est une totale innovation, dont les racines s'inscrivent dans le mouvement de contre-culture américain des années soixante.

Archéologie des pratiques

«Cette idée fut très lente à se dessiner, rien à voir avec une inspiration géniale! Tout a commencé en $1962 \ldots$ la première planche a été mise en chantier en janvier 1967. Je n'ai jamais cru à l'idée géniale ou à l'inspiration divine. Les idées c'est comme les fruits. Ça mûrit lentement et tombe de l'arbre une fois parvenus à maturité. C'est un processus très lent à se mettre en place.» (Jim Drake, Planchemag, août 1997).

5 En Europe, l'un des bassins de diffusion fut la Bretagne du Sud, autour de l'importateur avisé P. Carn. Jusqu'à la fin des années soixante-dix en France, le terme " Windsurfer " désigne un modèle de planche à voile (une marque), en même temps que les pratiquants; engins et acteurs sont intimement associés dans la dénomination. La marque Mistral connaîtra très vite une diffusion européenne qui supplantera Windsurfer.

6 La fin des années soixante-dix, sous l'influence des « régates Open » fera de la planche à voile un laboratoire d'expérimentations: architecture, matériaux, accessoires, etc. Toutes les possibilités seront déclinées, de l'industrie du plastique à l'artisanat du bois moulé, en passant par l'aluminium ${ }^{1}$. Des marques apparaissent spontanément, hors de toute considération de marketing. Certaines correspondent pourtant à la diversification de grands groupes industriels -chimie, plastique, intérim, presse...-, tels Sodim, Crit, Tornado, Ligier, Sainval, Océanite, Browning, VSD; toutes ces marques ont disparu, ce qui révèle de la part de ces groupes un intérêt conjoncturel et opportuniste. D'autres marques déclinent les savoir-faire de chantiers navals; c'est le cas de Dufour (qui deviendra Bic), Jeanneau, Feuillette, Alusurf (Wildfire), Fountaine, Pajot qui ont déjà investi le secteur du loisir nautique.

7 Enfin, de petites sociétés se créent : Tiger, Open Surf, Jet, Reix, Skipper (Magnum), P18, Naviplane, Grand raid, Seawolf, Alto, etc., des constructeurs allemands, suisses ou d'autres états européens -F2, Fanatic, Mistral parmi les plus célèbres- auxquels s'ajoutent des fabricants locaux, multiplient les moyens et les innovations.

«[...] Connaitre un peu mieux l'histoire des techniques permet de lutter contre cette vision un peu étroite des techniques sportives [...]. Quand on veut comprendre une invention, par exemple dans le domaine de la voile, c'est important de s'interroger sur le motif : qu'est-ce qui a poussé à agir, quelles sont les propriétés de l'environnement qui ont été utilisées? [...] Pour transporter des denrées, il a fallu qu'il y ait un volume dans les bateaux. Le problème, c'est que quand on donne du volume à une coque, elle devient moins performante pour partir en travers du vent. Donc il y avait une contradiction. Ça posait un nouveau problème et stimulait donc la nécessité d'inventer [...]. Le fait de ne plus avoir de denrées à transporter a redéployé, dans l'histoire de la navigation à voile, l'invention d'engins qui ont des performances extraordinaires actuellement pour remonter le vent, jusqu'aux planches à voile, où le bateau est réduit à sa plus simple expression. On ne peut pas beaucoup faire moins qu'un flotteur de planche de slalom, course, race, pour remonter au vent. Il y a juste de quoi supporter le pilote, et encore, si il s'arrête, il s'enfonce, et il y a juste de quoi l'empêcher de partir sur le côté. Et ça, ça va extrêmement vite, libéré des contraintes du transport, que l'on trouve encore dans 
le dériveur. Un dériveur, c'est encore un engin qui a gardé l'idée qu'on pouvait être dedans ou dehors. Alors est-ce que c'est un engin qui est destiné à disparaître ? Je n'en sais rien. » (Piegelin 1997-98).

8 À partir de 1980, le nombre des fabricants s'amenuise; seuls demeurent les deux géants nationaux (Bic, Tiga) et quelques shapers ${ }^{2}$ talentueux, en fabrication comme en gestion. $\mathrm{Au}$ cours des années soixante-dix, les utilisateurs sont autant créateurs que consommateurs; hormis pour la marque Windsurfer, les stratégies commerciales n'existent pas. Les principaux promoteurs de cette époque se nomment Hervé Borde, Patrice Valton et surtout Guy Ducrot, dont la formation d'ingénieur rompt déjà avec l'empirisme folklorique de mise dans la plupart des prototypes. Plus tard, Rénier Dobbleman, Yvon Piegelin, puis Hugues de Turckeim rationaliseront les prototypes Open. Dans ce contexte d'innovations, des formes et volumes délirants fleurissent : énormités, monstruosités, difformités, protubérances, excavations et boursouflures. Chacun y va de sa mégalomanie, exercée sur les flotteurs, mais aussi sur les voiles, dérives ou ailerons. L'arrivée de la jauge mettra un frein à ces expérimentations. Cela semble un processus général de l'innovation : l'esthétique est tout d'abord délaissée au profit de la performance, puis une autre génération d'engins intègre, en les épurant, ces avancées; la génération suivante les esthétise et les optimise. C'est ici aussi la collaboration entre coureurs et shapers qui a permis les progrès relatifs aux flotteurs et aux gréements. La planche à voile présente la particularité d'avoir été développée, expérimentée, optimisée par les pratiquants eux-mêmes.

La première génération de planches à voile n'a pas grand-chose à voir avec la glisse : les engins sont lourds et inesthétiques. Porter le flotteur (qui pèse parfois jusqu'à $30 \mathrm{~kg}$ ) nécessite une formation d'haltérophile et le chariot n'existe pas encore... Les véliplanchistes font donc de nombreux arrêts entre leur véhicule et l'eau; les plus courageux, en un mouvement proche de l'épaulé-jeté, soignent leurs biceps en calant le flotteur sur la nuque ou la tête. Ahanant, transpirant, rougissant, les véliplanchistes sont épuisés avant d'être sur l'eau. Et il faudra un second voyage pour le transport du gréement ! Ce dernier est coordonné : pied de mât en bois et inox, mât en aluminium ou en fibre, wishbone en teck (pour les Windsurfers), auxquels il faut encore rajouter une lourde dérive de contreplaqué ou de bois massif. Les voiles resteront blanches dans un premier temps, seul le logo de la marque y apparait; les " packs-écoles» des planches Windsurfers seront livrés avec des voiles orange. Les premières coupes de voiles sont plus proches du «spi de vaurien usagé » que des formes actuelles. L'esthétique d'ensemble est très discutable. Le polyéthylène est de mise, sa finition bosselée reste approximative. Le gréement est loin d'être élancé; il ne constitue encore pas une véritable rupture avec celui des dériveurs. Les laizes des voiles sont horizontales et ajoutent au sentiment de lourdeur, -la voile est d'ailleurs qualifiée de " poche ». Même les voiles-tempêtes des Windsurfers conservent la longueur standard du wishbone; elles sont coupées à partir du premier quart du mât.

Quant aux tenues des véliplanchistes, les premières combinaisons sont empruntées aux plongeurs : noires, épaisses de $6 \mathrm{~mm}$, en deux parties, et munies d'une cagoule. Les photographies, voire les publicités, des premiers numéros des revues Planche et Windsurfing (mais aussi de l'ensemble des revues nautiques, car toutes se sont saisies du phénomène et ont leurs rubriques "Essais et conseils») présentent donc une esthétique particulière du véliplanchiste: arc-bouté pour résister à la traction de la voile, il écarte les bras le plus possible, et bien entendu les jambes pour rechercher un semblant de stabilité. Une variante sera proposée plus tard lors des régates open sur les 
planches rondes; sur le bord de vent arrière, les partisans de la position accroupie, jambes et bras écartés, seront concurrencés par ceux de la position assise, les pieds dans l'eau, qui abaissent encore davantage le centre de gravité.

11 La planche à voile emprunte aussi beaucoup aux dériveurs classiques: la dérive, l'aileron qui, à l'origine, a une fonction stabilisatrice... Les inventeurs Drake et Schweitzer reconnaissent aussi un ensemble d'influences, dont celle des «Malibu», évolutions des surfs en bois massif. Hésitant encore à une autonomie totale, les fabricants de voiles incorporeront assez lentement la couleur

La planche à voile à ses débuts, est une affaire d'« hommes forts ». Les pionniers sont généralement issus du dériveur; formés à l'école de voile traditionnelle, ils se tournent vers la planche, car il n'existe quasiment pas d'alternative solitaire (le Finn est inaccessible, le Moth Europe confidentiel, le Laser encore inconnu) ${ }^{3}$.

Pendant quelques années, la promotion de la force ne s'accommodera pas d'un accessoire alors jugé superflu, le harnais. Son introduction sera laborieuse mais reléguera à la préhistoire la position de bras crochetés sur le wishbone. Sans le harnais, affronter 20 nœuds avec une Windsurfer relève de la prouesse. C'est d'ailleurs l'argument sécuritaire qui l'imposera... il permet en effet la disparition des crampes. Le harnais deviendra très vite une pièce constitutive de la panoplie des véliplanchistes, au point qu'il est aujourd'hui impossible d'en observer sans ce crochet vital.

Cette généralisation a induit une transformation complète de la technique et donc de la posture. On passe d'un système « vertical » issu du dériveur -où la première révolution réside déjà dans le passage de la position assise à la position debout- à un système « horizontal » caractérisant le funboard.

«Les nouvelles attentes vont d'ailleurs aux pratiques relaxées, aux gestes contrôlés et "mentalisés": tous ces exercices promouvant l'ajustement perceptif, le relâchement travaillé, ces jeux de pilotage et de glisse des nouveaux engins sportifs, surfs, ailes volantes, skis ou planches à voile, où le travail du sens peut l'emporter sur celui des muscles. » (Vigarello 1998).

15 La navigation sur la génération des planches fossiles promeut une culture de traction sur la voile; sur les planches de la seconde génération, on passe à une culture motrice de suspension, que permet le harnais; l'équilibre et le positionnement du centre de gravité sont essentiellement régulés par les membres inférieurs. Avec l'apparition de matériaux composites, tout au moins la généralisation des fibres, taille et poids moyens des engins vont fondre. L'ère du « light » va ici aussi s'imposer, parfois même confiner à l'éthique anorexique: on passe du «paquebot» de 3,90 m et $22 \mathrm{~kg}$ à la «fusée » de $2,50 \mathrm{~m}$ et $12 \mathrm{~kg}$... que peu de pratiquants réussiront à dompter.

16 À partir de cette inflexion, la pratique de la planche à voile peut être qualifiée de sport de glisse. La position d'équilibre dynamique, en propulsion, n'entretient aucune relation avec celle de l'équilibre statique : comme pour la bicyclette, c'est la vitesse qui accroît la stabilité : l'équilibre dans le couple engin/corps (poussée de la voile combinée à la force antidérive/corps et poussée d'Archimède) permet une glisse confortable ${ }^{4}$.

Les écoles de voile proposèrent des stages de planche sur le modèle de la "filière voile " de l'époque: pratique et théorie de la poussée vélique s'avéreront bien vite antinomiques de la culture du plaisir immédiat permis par des engins que l'on croit accessibles. C'est ainsi que les écoles de voile devinrent au début des années quatrevingt des centres nautiques, favorisant la démocratisation autant que la diffusion d'une 
«culture nautique». Mais le pas de l'autonomie ne sera jamais franchi avec la Fédération Française de Voile. Cette aspiration scissionniste qui se pose dans des termes proches lorsqu'il y a de nouvelles pratiques inventées, pour toutes les fédérations, a aujourd'hui été oubliée de la mémoire collective. Lors de la seconde moitié des années soixante-dix, l'influence de la planche (et du fluo) ira grandissante dans la société, davantage qu'au sein des institutions sportives. Elle permettra l'introduction de la couleur dans des milieux sportifs éminemment conservateurs, le tennis et le cyclisme. La sportivisation de l'activité favorise aussi les innovations technologiques; le recours successif aux fibres composites pour les flotteurs comme pour les voiles diffusera de la planche vers le nautisme traditionnel : carbone, mylar et autres kevlar, puis monofilm, feront d'abord leurs preuves sur des modèles de taille modeste. On peut même penser que l'architecture navale bénéficiera de certaines expérimentations pour oser extrapoler aux luges géantes que sont les maxis open...

Le prix d'une planche à voile est resté relativement stable au fil des ans par rapport à d'autres engins de loisir. Mais l'ensemble des activités nautiques demeurent cependant particulièrement onéreux. Diverses propositions favorisent pourtant leur accessibilité. La question complexe de la démocratisation des pratiques nautiques doit intégrer celle, déjà délicate, des coûts, mais également celle des "jeux sociaux", ou encore celle, culturelle, du rapport au milieu. Aujourd'hui, parmi les représentations dominantes figure celle héritée de la vulgate américaine des années cinquante: les théories de Maslow, et leurs arborescences, traitent des besoins des individus dans un cadre relevant de la psychologie sociale. Les besoins seraient hiérarchisés, des plus fondamentaux (organiques), aux plus facultatifs (estime, réalisation). Un besoin "supérieur " ne pourrait être satisfait que si les "précédents» le sont. Cette théorie des besoins, encore dite pyramide de Maslow, a été interprétée et traduite en termes sociaux pour donner corps à une théorie des motivations toute aussi hiérarchisée. Là encore, l'individu satisferait successivement des désirs de plus en plus élaborés. Ces théories présentent un danger de simplification; leur logique déterministe et mécaniste masque les complexités psychologiques et sociales des acteurs. Les pratiques de loisir ne sont pas réservées à ceux qui ont épuisé les motivations non ludiques... aujourd'hui encore moins qu'hier! La lecture, la musique, le sport... peuvent constituer des passions qui motivent tant il n'est pas rare d'observer des acteurs -ou des ménagesqui gèrent leur budget de façon apparemment « irrationnelle ».

« Depuis que j'ai découvert la planche, tout mon argent y passe. Ça a commencé à 16 ans, les cadeaux et anniversaires, aujourd'hui tout y est consacré. J'ai une poubelle comme voiture, je ne fais pas de folie pour la bouffe, le plus souvent au Resto-U ou chez mes parents, je bosse un peu... mais j'ai du matos top. Il y a aussi les combines pour avoir des réduc, savoir quand acheter...»

Le facteur culturel concerne le rapport au « milieu naturel » et à l'environnement. Les pratiquants de loisir actuels, qu'ils soient réguliers ou saisonniers, s'initient à l'activité et découvrent en même temps le milieu marin.

Le divorce sera très vite consommé entre une formation "maritime " puis sportive, et une formation ludique et sportive, où dans le meilleur des cas, le milieu sera appréhendé par essais-erreurs successifs. Météorologie, marées, courants, sécurité, peuvent ainsi être considérés comme des phénomènes accessoires (ou négligés) par de nouvelles générations de pratiquants, jeunes et adultes. Ce qui explique pour partie les accidents. Les véliplanchistes sont souvent dénigrés, comme le sont en montagne les surfeurs des neiges. Les arguments sont d'ailleurs proches: vitesse, dangerosité, 
méconnaissance des lois de la nature... Les formateurs conservateurs du nautisme les stigmatisent sur un registre didactique :

«Ils ne savent rien de ce qui se passe sur l'eau, ils tirent sur le wishbone et bourre, bourre..., ni tactique, ni météo, ni technique, ça fait beaucoup quand même par rapport au dériveur. C'est un peu comme la génération de catamaran fun... on se pose pas de question!»

21 Au-delà de leur formulation lapidaire, ces critiques sont également à entendre dans la double compétition que se livrent les générations entre elles, mais surtout celle des groupes sociaux entre eux. Il en va de l'accès à la mer, de l'appartenance au monde maritime, que bien souvent les mêmes qui s'attachent à en proclamer la nécessité, refoulent aussi avec le même acharnement. La réflexion sur la démocratisation des sports nautiques doit se saisir de cette problé-matique. Les véliplanchistes revendiquent une inscription dans "la famille" de la mer; des signes en sont perceptibles lors, par exemple, de l'hommage au marin disparu Éric Tabarly, Planche Mag consacre (dans son numéro d'août 1998) un article intitulé « Un grand homme nous a quitté ", tout en rappelant le souvenir des martyrs de la tribu planche, eux aussi disparus en mer, eux aussi aspiraient à une soif de liberté. Ainsi que l'écrit Jean Rieucau (1997 : 149).

«La mer apparaît donc dans ces conditions comme le dernier bastion contre les avancées d'une civilisation qui réduit l'autonomie des individus [...]. Le mythe d'une mer éternelle, infinie devient indispensable. Il permet à l'individu de s'évader réellement ou en rêve et de reconquérir l'image d'un conquérant acteur de son destin. »

L'univers de la planche à voile porte aussi les traces d'autres paradoxes. Les rebelles forment des marginalités recherchées - pour en faire des « sujets »- par les magazines spécialisés; les antisportifs revendiquent des trajectoires hors normes, comme les réfractaires au « système » peuvent l'exprimer dans certaines revues de rock.

La première décennie de la planche en France (entre 1975 et 1985) a davantage été marquée par les héros que par les sportifs. Les traversées en solitaire se succédaient: Marty, Peyron, Beauchêne écrivent la légende des aventuriers, R. Naish et K. Winner celle des sportifs. Qui se souvient toutefois que les compétitions de planche à voile sont alors bien souvent de grands rassemblements qui donnent lieu à des épreuves d'endurance ? Les 18 heures, les 24 heures de..., le tour de... On est bien loin d'une pratique " fun "! À son début, la planche à voile oscille entre l'innovation véritable et un ensemble d'influences, notamment nautiques, dont elle veut pourtant se démarquer. Une esthétique balnéaire

24 Lorsqu'à la fin du XIXe siècle, les plages bretonnes sont envahies par les touristes anglais et les villégiateurs français, la révolution corporelle, et donc culturelle, se met en marche. Le chemin de fer contribue à ces nouvelles migrations.

Comme hier, quand les nouveaux usages de la plage furent définis par ces voyageurs étrangers apportant alors le lawn-tennis, le diabolo, le golf..., aujourd'hui, apparaissent des activités nouvelles. Le cerf-volant, la planche à voile, le beach-volley, transforment, non sans heurts parfois, l'esthétique balnéaire.

La planche à voile constitue aujourd'hui un révélateur intéressant. Les véliplanchistes sont des funboarders. Les termes témoignent d'ailleurs de l'appar-tenance à une génération: au début de la decennie quatre-vingt, aux pan-am des in and out, ont succédé les guns de vitesse inspirés du surf; eux-mêmes relégués par la génération des 
raceboards et autres slaloms ou waves; les formes du flotteur sont scoop et outline; les pin tail et no nose du début des années quatre-vingt-dix sont déjà hors jeu; le même sort guette la génération freeride et convertible, puisqu'apparaissent les freerace.

Les flotteurs sont devenus légers, en même temps que les voiles se faisaient transparentes et que les silhouettes s'affinaient... Les marques et les revues de planche préfèrent bien entendu les hawaiiens longilignes aux petits gros, quadragénaires grisonnants. L'apparence de jeunesse et de facilité prime sur le style bûcheron.

La motricité du véliplanchiste fun renvoie à une éthique de plaisir issue de sensations et d'esthétiques. La pureté, la fluidité, la placidité, la douceur, la limpidité, sont les figures de l'eau sublimée alors que l'adversité, la violence, la douleur, la colère, la furie, sont celles de l'eau dangereuse. Chez le planchiste comme chez le nageur coexistent pourtant des sensations aussi éloignées que le bien-être et la souffrance.

«[...] l'ambivalence spéciale de la lutte contre l'eau avec ses victoires et ses défaites s'insère dans l'ambivalence classique de la peine et de la joie [...] le sadisme doit faire place tôt ou tard au masochisme. » (Bachelard 1996 : 206).

Les discours passent le plus souvent sous silence les blessures, les ampoules, les chocs qui peuvent y apparaître toutefois, mais euphémisés; c'est alors « la gamelle », «le soleil », « la lessiveuse »... La quête de l'extrême et la traque du danger ne font pas partie du décor; la métaphore militaire n'est pas au rendez-vous, malgré l'ambivalence des sentiments.

Bien avant la navigation proprement dite, une multitude d'activités préliminaires s'inscrivent dans cette culture de l'aisance naturelle: l'arrivée en véhicule, le déchargement du matériel, son gréage, la mise en tenue, le transport et la mise à l'eau ne procèdent aucunement du hasard :

«Ça se travaille, au premier coup d'œil, tu sais à qui tu as à faire; déjà le matos te donne des indications, soit il est vieux, soit c'est dans le coup; a priori, sur un spot, on se connaît plus ou moins [...]. Tu regardes le mec, comment il pose ses affaires, comment il s'y prend [...] mais l'air de rien quand même, pas mateur... »

31 Les acteurs de la plage jouent tous leur partition de la chorégraphie collective: discrétion, ostentation, évitement... tous les comportements coexistent.

« La plage n'est qu'un vague agrégat de personnes toutes différentes, irréductibles à une pensée unique [...]. Elle a réussi à constituer son gouvernement, à ériger des règles molles mais contraignantes, à imposer une idéologie latente [...]. Mais un petit fragment de conformité ajouté à une infinité d'autres petits fragments suffit pour que se dégage une pensée collective dans la diversité multiforme des vies particulières. Ce rivage est un lieu de grande liberté [...]. L'individu est face aux éléments naturels, dépouillé de la plupart des oripeaux de la civilisation, seul avec son corps, plus près de lui, enveloppé de sensations épidermiques [...]. La plage est réellement ainsi en surface. Mais elle est également, tout aussi réellement, différente en dessous, où un autre monde se révèle, dans lequel rien n'est laissé au hasard. » (Kaufmann 1995 : 127).

Réduire les véliplanchistes au qualificatif de «frimeurs" témoigne d'une méconnaissance de l'auto-régulation des comportements humains. L'un des pères de l'interactionnisme pointe leur mise en jeu scénique en l'absence même d'autrui :

« Dans ce cas, l'acteur en arrive à être son propre public, à être tout à la fois acteur et spectateur du même spectacle, c'est sans doute qu'il intériorise et assimile les normes qu'il s'efforce de maintenir en présence d'autrui, à tel point que sa conscience l'oblige à agir d'une façon socialement acceptable [...]. En d'autres 
termes, il est possible à un acteur d'être son propre public ou encore d'imaginer la présence d'un public.» (Goffman $1987: 18$ ).

L'appartenance à un groupe passe par l'imprégnation consciente ou non des codes et gestuelles qui agrègent ses membres. C'est « l'autrui significatif » qui participe de la socialisation motrice et esthétique des tribus de planchistes :

«L'individu intègre dans son image de lui-même ce qu'il connaît de son apparence, directement ou par les réactions de ses partenaires [...]. On n'exprime pas, pour l'apparence, n'importe quoi, n'importe où, n'importe comment et dans n'importe quelles conditions. Il existe une sorte d'éthique de l'apparence qui constitue une autre forme d'intégration de celle-ci au milieu sociologique.» (Duflot-Priot 1987 : 11).

"L'effort ne doit jamais être visible [...]. La technique doit être entièrement camouflée. Ni l'arrivée d'un saut, ni la fin d'une pirouette, rien ne doit être brusqué, tout doit être retenu. " (Lacotte 1990).

Le détournement vaut d'être signalé car la citation traite de la danse classique et du ballet, et non de la planche à voile. Ici aussi, la « légèreté suggérée exprime la qualité de ce qui a peu de poids, ce qui est aérien, qui effleure, évoquant la maîtrise du geste et la délicatesse de la réalisation. La légèreté s'oppose à la lourdeur (au sens propre et au sens figuré) » (Arguel 1996 : 7).

La fluidité du mouvement, l'invisibilité de l'effort, la légèreté se retrouvent dans la génération des nouvelles pratiques corporelles ou dans les dérivés d'acti-vités sportives. Le défi à la pesanteur s'exprime dans les sports de glisse, de grimpe, de vol mais aussi dans des hybrides de danse et de sport tels la danse-escalade ou la danse voltige ${ }^{5}$.

Pourtant, l'élasticité de la silhouette, la fluidité de la démarche et la facilité apparente de l'action ne sont pas des dons naturels lorsqu'il s'agit d'emmener son matériel au bord de l'eau dès qu'il y a du vent. Si flotteurs et gréements ont largement été " dégraissés ", la plupart des ensembles sont inférieurs à $15 \mathrm{~kg}$; leur prise au vent est, elle, constante. Comment dès lors rendre compatible le culte de l'apparence hédonique et l'exercice de force?

« Tu as bien les papys de quarante ans qui utilisent encore des chariots, mais aujourd'hui, ça ne se justifie pas... En plus, c'est doublement galère, car suivant l'heure de la marée, t'es obligé de faire encore un autre voyage pour le stationner [...]. Il y a aussi le risque de se le faire faucher. Parfois on voit des petits jeunes qui n'ont pas le permis et qui habitent pas trop loin arriver avec leur chariot, à pied, ou même derrière un vélo ou une mobylette... Faut être motivé ».

Le chariot est l'accessoire ventru du véliplanchiste; il est en quelque sorte perçu comme la verrue de la panoplie dans la perspective énoncée par notre informateur, pourtant doté, lui, d'une voiture et d'une remorque à planche...

La descente du matériel est un acte important. Les moins expérimentés peuvent « assurer » en descendant flotteur et gréement en deux temps, voire en gréant au bord de l'eau, ce qui limite les risques.

« Ta planche, tu la prends sous le bras et tu descends tranquille; les jours où il y a beaucoup de vent, c'est pas si simple, ça prend dedans, et tu peux très bien tituber dans tous les sens si tu ne sais pas trop y faire... Pour le gréement, c'est pareil, tout dépend de la direction et de la force du vent, c'est comme sur l'eau...La voile doit présenter le moins de prise au vent, sinon, c'est pas la peine... Le top c'est de descendre en un seul voyage, flotteur et voile gréée; c'est assez technique, mais là... ». 

footstraps du flotteur d'une main et le wishbone de l'autre, ce qui serait relativement simple sans vent; la difficulté réside dans le déplacement vers l'eau en présence d'un vent qui est tout de même la condition sine qua non de la navigation... Si l'exercice demande de l'entraînement, c'est aussi parce que le plus haut niveau d'expertise doit s'accommoder d'une certaine nonchalance, d'un détachement ostentatoire de ces contingences matérielles. Ici, « l'air naturel » nécessite une maîtrise totale de la culture technique. Les réajustements à l'orientation et à l'intensité du vent sont permanents... la démarche doit, elle, rester souple et rectiligne. Imaginez un instant le paradoxe du regard scrutant l'horizon, du sourire détaché... et de muscles tétanisés par l'effort, une concentration totale sur les variations du vent. L'exercice est ici assez proche de celui du culturiste posant sur scène, lors d'un effort total... mais le sourire aux lèvres.

Pour s'être risqués, en l'absence de maîtrise complète, à tenter de transporter tout le matériel (planche et gréement) -ainsi que le mettent en scène les photographies de la presse spécialisée-, certains se sont retrouvés par terre, la voile ayant pris «à contre ». Là, point de salut lorsque le vent s'oppose :

"Il vaut mieux tout lâcher, tu peux alors te récupérer, tu empannes, c'est comme sur l'eau... C'est toujours le cas lorsque tu rentres de nav', d'abord tu es fatigué par l'effort, et en plus, lorsque tu arrives près du parking ou de la digue, le vent tournoie dans tous les sens, alors là, c'est pas la peine, ça va pas le faire... ».

connait donc les «blaireaux » à ce premier exercice de la descente de plage... et non pas au temps qu'ils mettent à gréer. Car les "pros " ${ }^{6}$, eux, évaluent parfois longuement les éléments météorologiques, avant et après la navigation. La grande question du parking est celle de la bonne surface de voile; il s'agit de ne pas se tromper; alors discussions et palabres vont bon train.

« Tu mets combien ?, Et X, il a pris quoi ?, Ouais la 5,5 $\mathrm{m}^{2}$ ça paraît pas mal, mais au fond, ça a l'air de forcir, alors j'hésite... Je vais peut-être prendre petit car je reste naviguer tout l'après-midi, et puis là-bas, ça forcit avec l'effet venturi, je compte rester par là... ».

ésitations durent parfois l'après-midi entier... après, il est trop tard pour aller sur l'eau. Des observations estivales relèvent ainsi des groupes consé-quents de planchistes préparant et gréant tout le matériel vers midi, en attendant le vent qui ne viendra peut-être pas: " Ici tous les jours le thermique se lève, il vaut mieux être prêt; comme ça on prend son temps, on discute... ». De fait, il arrive des exceptions à la régularité du vent thermique; c'est ainsi une journée consacrée à transporter, décharger, gréer, guetter, dégréer, ranger... et surtout palabrer avec sa tribu. Les rationalistes ont eux consulté la météo, par téléphone, Minitel, journal, etc., certains le font toute la semaine et guettent l'arrivée de la dépression, organisent leur emploi du temps et prévoient une demi-journée de congé.

D'autres pragmatiques ne se déplacent qu'avec un anémomètre; la force réelle du vent est mesurée et définit la surface de voile adéquate. Parfois quand même, certaines prises de mesures feraient sourire les météorologues... un parking, le haut d'une digue peuvent parfaitement biaiser la force du vent sur l'eau. Dans la majorité des cas, la prise d'informations est complexe; elle renvoie au croisement de tout un ensemble d'indices fournis par les éléments naturels ou obtenus des véliplanchistes qui naviguent déjà.

Techniques \& Culture, 39 | 2002 

particulièrement délicate, celle de la mise à l'eau. Bien souvent, l'exercice n'est pas aussi simple qu'il y paraît. Le beach start exige lui aussi des compétences aussi élaborées que celles requises pour la navigation: «De toute façon si tu ne réussis pas le beach start, c'est pas la peine d'essayer le water start ». Le départ dans l'eau procède lui aussi de ce bagage technique. Qui n'a pas pourtant observé l'activité du débutant faite de chutes répétées, d'immersions prolongées, de chocs incessants! Ce spectacle fait désormais partie intégrante du paysage balnéaire estival. Rares toutefois sont les informateurs qui reviennent spontanément sur un apprentissage souvent idéalisé, esthétisé; « ça a été assez facile » est le motif récurrent... Faire référence à une école de voile, un centre nautique, à un "apprentissage non spontané ", à des difficultés, ne relève pas des discours les plus courants.

Une culture du vertige

«La nouvelle mythologie sportive ne met donc pas seulement en scène un individu qui soigne sa forme et son apparence dans une société où la jeunesse est une norme et non plus une classe d'âge, il forge de l'individu, un individu héroïque qui prend des risques au lieu de chercher à s'en protéger par les institutions de l'ÉtatProvidence, qui cherche à agir de lui-même au lieu d'être commandé par d'autres. » (Ehrenberg 1996 : 186).

Plutôt que de la définir comme une essence supposée et figée, la culture, dans une perspective interactionniste, est une construction émanant du social. « Le véritable lieu de la culture, ce sont les interactions individuelles ». Il en va des contre-cultures comme des subcultures; elles constituent des sous-ensembles qui contribuent à renouveler et développer la «culture globale de référence à laquelle elles sont censées s'opposer » (Cuche 1996 : 130).

On a déjà souligné l'existence d'arborescences culturelles: les compétiteurs, les sportifs, les waveriders, les randonneurs... Outre les différentes façons de faire, des 
caractéristiques distinguent ces sous-groupes : territoires, matériels, codes langagiers, vêtements... Le paradigme transcendant les discours porte sur le vertige et le risque. Entendons-nous : davantage que de dangers objectivables, il s'agit d'une prise de risque fantasmé au regard d'un niveau de pratique. Pour l'essentiel, elle pose la chute comme sanction. Le défi, la lutte contre les éléments et contre soi-même animent les discours.

Le contrôle et la maîtrise de l'engin apportent "l'éclate", "la défonce "; c'est un rendez-vous avec soi-même, une épreuve renouvelée. Ici, le plaisir de la situation et des sensations caractérise au plus haut point «l'esprit fun». R. Caillois distingue quatre catégories dans les jeux: l'aléa (le hasard), l'agôn (la compétition), la mimicry (le simulacre) et l'illinx (le vertige). Il est toutefois difficile d'isoler les principes en œuvre dans l'action de glisse :

« [L'illinx relève des jeux qui] reposent sur la poursuite du vertige et qui consistent en une tentative de détruire pour un instant la stabilité de la perception et d'appliquer à la conscience lucide une sorte de panique voluptueuse. Dans tous les cas, il s'agit d'accéder à une sorte de spasme, de transe ou d'étourdissement qui anéantit la réalité avec une souveraine brusquerie » (Caillois $1967: 67$ ).

51 Les véliplanchistes qualifient ces instants confus de vertige, de contrôle et de victoire sur soi de « l'esprit fun », « le pied », « c'est cool », « difficile à expliquer ».

«La glisse conjugue également le sentiment du vertige avec celui du contrôle physique de la situation. Elle prodigue la dépense et l'ivresse et repose sur la confiance en ses ressources personnelles. » (Le Breton 1991 : 61).

Tout se passe comme si le plaisir provenait d'un contrôle de la situation, lui-même procédant d'un faible écart entre les capacités de l'acteur et l'action à réaliser. La victoire sur le danger peut ainsi être la chute évitée, la vague franchie, la navigation par une force de vent tout à fait aléatoire... Tout dépend de l'individu, du regard qu'il porte sur la situation ainsi que des représentations qu'il s'en fait. Il s'agit de chercher "la limite", nécessité anthropologique car vitale. La recherche du risque et de l'aventure ne va pas sans le paradoxe du contrôle social et de l'obsession sécuritaire. Chaque acteur intègre cette équilibre sécurité-risque. Le goût du risque est porté par l'éthique du dépassement et de la performance des sociétés occidentales. L'homme, et plus encore le jeune homme, ordinaire, cherche à s'éprouver, à remplir sa vie des émotions qui vont lui conférer du sens. Le risque, à mon sens et tout au moins pour ce qui concerne les véliplanchistes, n'est pas mortifère... l'observation participante s'oppose ici avec force à l'objectivation distanciée. Les pratiquants développent un instinct grégaire. Rares sont les véliplanchistes isolés.

"Je préfère naviguer avec mes potes, ça me donne des repères, on peut se mesurer, se tirer des bourres... Si tu casses, il y a toujours quelqu'un pour te donner un coup de main, appeler une sécu ou simplement pour tourner autour si tu galères. C'est pour ça que je navigue sur mon spot, sur deux ou trois spots maximum.»

Des observations régulières sur la Côte Nord (Bretagne) ou en presqu'île de Crozon attestent de navigations collectives, en petits groupes. Les sorties d'individus isolés, particulièrement dans des conditions de mer ou de vent difficiles sont trop marginales pour être signalées.

"Quand tu débarques dans un coin inconnu, tu cherches d'abord les locaux pour savoir où ils naviguent... C'est plus sûr... Livré à toi-même et sans connaissance ni des hommes ni des lieux, tu vas régresser, tâtonner, assurer... La prise de confiance ne vient qu'après une appropriation affective du territoire. » 
54 notamment dans les pratiques sportives, où la réassurance terrestre succède à la prise de risque nautique ou aquatique (plongée). Le frisson consécutif à une activité engendrant simultanément ou alternativement peur, plaisir et assurance qualifie les sportifs qui évoluent aux extrêmes. L'intensité des émotions est alors liée à la distance de dépassement de la zone de sécurité du sportif ordinaire. G. Azemar déclinait, lors des années soixante-dix pour les STAPS, la notion de «balance sécurité-risque » chez le jeune enfant, qui accroît progressivement sa zone d'activité, en présence de sa mère. Les familiers de l'extrême sont très rares en planche à voile; les pressions sociales et sécuritaires se chargent de limiter la prise de risque, pourtant nécessaire à l'apprentissage. À l'heure où le sens commun déplore, la perte de repères, la prolifération d'activités contraphobiques, c'est-à-dire « désirées bien que dangereuses et recherchées pour être maîtrisées, apportant un plaisir intense de sentiment de victoire sur soi » (Thomère 1995), répond aux angoisses fondamentales. "Le temps de l'épreuve reste une capitale du souvenir, l'un des moments-clés de l'existence et de la constitution du sentiment d'identité » (Le Breton 1997).

un registre plus sociologique, cette recherche de limites dans un corps à corps avec la nature, participe d'une possibilité de réenchanter le monde, voie tentante pour les jeunes. Ces perspectives sont tout autant vertiges qu'affrontements. Pour D. Le Breton (1991), la recherche du vertige est le fil conducteur d'une série d'activités qui connaissent un fort engouement, où l'individu se met imaginairement ou réellement en danger; mises en danger minimes et contrôlées la plupart du temps, mais au sein desquelles les accidents n'épargnent ni les hommes ordinaires, ni ceux d'exception : en alpinisme, escalade, vol à voile, voile, planche à voile... on ne compte plus les disparitions et accidents.

56 La recherche de la performance ou du vertige infère la nécessité de produire sa propre identité, de se prouver à soi-même la valeur de son existence, de conquérir la place à laquelle on aspire.

«Ça fait 20 ans que je fais de la planche à voile; quand j'ai débuté, on naviguait en groupe à 3-4 copains, en ayant vraiment l'impression d'être des aventuriers. Ca nous a permis de visiter la côte. Dès force 3 , on avait l'impression de naviguer dans du gros temps; à force $4 / 5$, on gréait la voile tempête sur la Windsurfer, difficile à tenir dans ces conditions de vent. Aujourd'hui, avec le même temps, on sort de grandes voiles entre 6 et $7 \mathrm{~m}^{2}$. Le matériel a beaucoup évolué, tout est beaucoup plus facile... Après, il y a eu la période régates, des déplacements de plus en plus longs, la recherche du meilleur matériel, l'entraînement, la performance, la compétition : on marquait des points pour son classement, pour celui de son club. Chacun gérait son classement. Malgré tout, on fait assez vite le tour de tout ça, alors à un certain âge, quand j'ai eu mon boulot et les enfants, c'est pas qu'on s'assagit, mais la compétition, c'était cuit et de toute façon, moins captivant... Le funboard est arrivé, ça a créé une nouvelle aspiration; on sortait dans des conditions plus coriaces, des vagues, on découvrait des sensations totalement nouvelles, de glisse, de saut, de vitesse. Dès que j'ai pu, en fonction du boulot, j'ai acheté une maison sur la côte, les pieds dans l'eau et à partir de laquelle je peux surveiller toutes les évolutions du plan d'eau. Être basé ici, c'est le rêve, même si tous les jours, j'ai 40 bornes pour aller bosser... J'ai des copains qui passent régulièrement, un autre ou deux qui laissent leurs matos dans le garage et viennent naviguer de temps en temps... Moi quand les conditions sont bonnes, j'hésite pas à poser un congé. Je continue à suivre les évolutions du matériel avec les revues ou avec un pote qui tient un magasin. Mes enfants s'y sont mis dont un qui régate un peu. Ils sont 
mordus, même si l'aîné préfère le surf. Pour les vacances, on choisit les spots ensemble; on retrouve chaque année des connaissances, que ce soit en Corse ou au Maroc. J'ai molli pourtant, même si je navigue toujours, je suis moins furieux, je veux pas me casser. Le fait de naviguer chez moi ou en groupe, ça me rassure aussi. Sur une saison de nav' il y a toujours quelque chose qui pète même si tu vérifies le matériel, un mât, un wish, une pièce ou une erreur et crac! Je compte plus les galères, tout seul à ramer pendant une heure, mais c'est pas la panique. Par moments je me dis qu'il faudrait passer à autre chose... Et puis tout s'organise autour de la planche, alors c'est pas demain que je vais m'asseoir pour regarder les autres naviguer. »

Ce technicien d'une quarantaine d'années est domicilié dans le Finistère; arrivé depuis une douzaine d'années à la suite d'une mutation, il a privilégié le cadre de vie et sa passion sportive. Son intégration s'est réalisée davantage par son loisir que par sa profession, vécue comme accessoire et nécessaire. Son statut principal n'est pas ici indexé sur la profession et sa trajectoire ne s'inscrit pas dans une logique de promotion sociale. Sa revendication du statut de véliplanchiste est permanente; la référence nautique ou maritime n'est pas présente. Ici comme ailleurs, l'identité collective se construit par rassemblement et opposition: le groupe des surfeurs semble présenter une proximité ou une filiation plus revendiquée que celle des plaisanciers, par exemple. Le groupe de référence, celui des planchistes, fournit à notre informateur des valeurs, des normes, des attitudes, des codes vestimentaires. Les réseaux de sociabilité favorisent également le regroupement local; celui-ci est constitué de familles qui occupent le même territoire géographique, il permet de nombreuses coopérations et relations, entre adultes mais aussi entre enfants. "Tuyaux et bons plans» sont échangés. Des solidarités transgénérationnelles ou professionnelles sont déclinées.

D'autres acteurs trouvent leur motivation dans la compétition; nos propres travaux montrent aussi des translations de motivation, y compris dans une même pratique sportive, à mesure qu'on avance en âge. Ainsi, le véliplanchiste, comme les autres sportifs, peut successivement rechercher la griserie, la compétition contre les pairs, la navigation avec les amis, l'exercice de freestyle, la prise de risque, le maintien de la forme, l'appartenance à un groupe, etc. Il en va de la planche à voile comme des autres activités sociales (Lacombe et al. 1995).

59 La sportivisation de la planche à voile est peut-être une fausse évidence. Alors qu'aux origines la plupart des adeptes s'affrontent lors de différentes joutes qui sont autant des courses que des fêtes, la pratique régatière est aujourd'hui quasi confidentielle. Les différentes variétés (open, longboard, slalom, vitesse, vagues...) sportives n'ont pas facilité la cohérence de l'ensemble.

Les épreuves sportives sont devenues quasi exclusivement des régates : certes il existe les épreuves de vitesse, les affrontements de vagues; elles ont en commun de ne concerner que quelques rares élites ${ }^{7}$. La pérennité des compétitions sportives passe au moins par deux conditions : pouvoir être suivies par un public non spécialisé et être restituables par les médias télévisuels. L'intelligibilité des épreuves suppose leur simplicité. Les épreuves olympiques de planche à voile sont aujourd'hui aux épreuves de vagues ce qu'étaient les régates de yachtmen aux courses de bateaux de pêche... ces dernières étant suivies au début du siècle, par des milliers de spectateurs.

61 On peut d'ailleurs se demander comment l'aspiration à la nature, la soif de liberté, la quête du plaisir peuvent s'accommoder de l'éthique compétitive. Cette motivation est 
rarement exclusive, même si au plus haut niveau, la souffrance est nourriture quotidienne. Deux champions du monde de vitesse l'expriment lors d'entretiens :

«À Brest, quand on court, le premier ennemi c'est le froid... Aux Canaries, on a navigué 10 jours d'affilée, 10 jours de soleil, de brûlures, de baston... mains arrachées. Dans les deux cas, le plaisir reste intact, encore faut-il que le corps soit préparé ».

" Aux Canaries, pendant 10 jours, 4 à 5 heures par jour sur l'eau... on a chaud, on est déshydraté. Je fais ça pour gagner ma vie, alors le reste... C'est comme les pêcheurs, moi ma satisfaction, c'est la performance, c'est pas de la plaisance... »

D'autres compétiteurs l'expriment également :

« En athlétisme, en danse ou en planche, on souffre et on s'amuse, on a mal et on prend du plaisir... Il n'y a pas de sports masochistes ou uniquement hédonistes. Entraînement et compétition, les deux dimensions alternent parfois le même jour, la même minute, le même instant... "

Mais la dimension de dépassement est permanente nous l'avons vu, affrontement à soi et à l'autre.

"C'est bien simple, sur ce spot, tu peux pas mettre à l'eau sans que tout de suite tu sois branché... T'as 10 minots qui ne rêvent que d'une chose, c'est de pouvoir te mettre d'équerre..., alors que toi, tu veux naviguer, eux ils rêvent de pouvoir dire "t'as vu ce que je lui ai mis à X". »

Les conflits d'usages sont rares mais sur un «spot urbain » comme la rade de Brest, la surpopulation rend parfois la sortie périlleuse. Une observation des secteurs de navigation montre toutefois des répartitions territoriales très marquées.

«Regarde bien, tout le monde fonctionne pareil, tu navigues dans le même coin : le premier rideau c'est au bord de la plage, le second c'est ceux qui assurent à 200 mètres du bord, ils font les mêmes aller-retour, après tu as le secteur entre le pont et Keraliou, enfin tu as au fond le polder et Kernisi... Je crois que c'est par niveau à peu près homogène, des débutants à celui des experts. Le seul risque c'est le départ et l'arrivée de plage où ça n'assure pas trop!»

Glisse, nature, liberté

Différents auteurs ont proposé des cadres théoriques restituant les pratiques de glisse :

«Il semble bien qu'une ligne de partage forte passe entre pratiques organisées et pratiques informelles, pratiques de compétitions officielles, privilégiant les valeurs de concurrence, de performance, d'émulation, et pratiques de démonstration mettant davantage en avant les valeurs de coopération, d'entraide, de solidarité. Si l'on suit cette analyse, deux types de sportifs se dégagent: les sportifs "traditionnels" respectueux des règles et disciplines édictées par leurs instances fédérales, les "nouveaux sportifs" ou sportifs "fun", créant leurs propres règles, s'autodisciplinant. » (Fize 1982 : 168).

Nombreux sont les sociologues du sport à s'être attachés à la construction de cadres théoriques permettant de restituer les mécanismes complexes et antagonistes au sein des pratiques sportives. Pierre Parlebas propose d'en restituer la logique interne à partir des systèmes de communication motrice (avec le partenaire, l'adversaire, le milieu). Christian Pociello s'attache aux caractéristiques des pratiques dans la relation qu'elles entretiennent à l'habitus des groupes sociaux. Plus récemment, Alain Loret restitue le détachement des néo-pratiquants envers la traditionnelle pratique au sein de la Fédération :

«Les sports digitaux sont des sports d'exclusion pratiqués dans un objectif de reconnaissance de soi. Cette reconnaissance prend la forme d'un rang au sein d'une hiérarchie attribuant de ce fait un statut particulier à celui qui s'y positionne. Ce 
statut correspond à une identité sportive, mais aussi sociale, qui prend le pas sur la propre identité du champion. Les sports analogiques sont, au contraire, des sports d'inclusion que l'on pratique simplement dans un objectif de connaissance de soi. La participation du plus grand nombre est leur raison d'être car la production de chiffre n'est pas leur objectif. Ils ne rendent compte d'aucun statut particulier, ni d'aucune hiérarchie à l'intérieur de laquelle les individus pourraient se faire reconnaître. Lorsque l'on s'engage dans une pratique sportive analogue, c'est pour savoir ce dont on est capable, pas pour le montrer. La performance, si performance il y a, sera connotée et non pas dénotée. C'est-à-dire qu'elle n'aura pas de signification par rapport à une référence qui lui serait extérieure. Elle aura, par contre, un sens apprécié de façon entièrement personnelle. » (Loret 1996 : 231)

Les activités sportives de loisir ne sont pas accessoires ou futiles. Elles structurent le rapport au monde des pratiquants, dont les sociabilités et modes de vie témoignent au contraire d'une place centrale, parfois. Les identités collectives, locales, groupales ou tribales, peuvent être conférées par une pratique ou ses modalités. Elles sont en effet plurielles, multiformes et en perpétuelle évolution; à l'image des surfers, les temps, lieux et matériels des véliplanchistes symbolisent des compé-tences techniques ainsi que l'appartenance à une famille. La plupart du temps, le sport de glisse n'est que rarement un sport à risque. La vitesse et l'« éclate " structurent davantage les discours et les représentations que les pratiques pour les pratiquants observés et entendus; cette mythologie de la mer et du risque est importante, mais ne confère pas mécaniquement l'appartenance à une culture maritime ou écologique.

\section{BIBLIOGRAPHIE}

Arguel, Mireille

1996 « Paroles de danseurs, paroles de chercheurs », Autrement 164 ("Légèreté »).

Bachelard, Gaston

1993 L'eau et les rêves. Paris : éditions J. Corti.

Caillois, Roger

1967 Les Jeux et les hommes. Paris : Gallimard.

Cuche, Denys

1996 La Notion de culture dans les sciences sociales. Paris :La Découverte (« Repères »).

M.T. Duflos-Priot

1987 « L'apparence et son bon usage », Les Cahiers du LERSCO.

Ehrenberg, Alain

1996 Le culte de la performance. Paris : Éditions Pluriel (1ère édition 1993).

Fize, Michel

1982 « Les pratiques sportives et leurs modes d'expression », in Sport, relations sociales et action collective.

Goffmann, Erwing B. 
1987 La Mise en scène de la vie quotidienne. Paris : Éditions de Minuit.

Kaufmann, Jean-Claude

1995 Corps de femmes, regards d'homme. Paris : Nathan (« Essais et Recherches »).

Lacombe, Philippe et al.

1995 Vers un observatoire des Pratiques Sociales et Sportives à Rennes. UFRAPS, Université de Rennes 2.

Lacotte, $\mathrm{P}$.

1990 Art de la danse de G. Mannoni. Paris : Plume-Calmann-Lévy.

Lafourcade, Georges

s. d. La Jeunesse de Swin burne. Tome 1.

Loret, Alain

1996 "Génération glisse : dans l'eau, l'air, la neige... la révolution du sport des "années fun" ", Autrement 155-156 (« Mutations »).

Le Breton, David

1991 Passions du risque. Paris : Métaillié.

Piegelin, Yvon

1997-98 Les carnets des STAPS nº1. Université de Bretagne Occidentale.

Rieucau, Jean

1997 « Sociétés maritimes et sociétés littorale : quelle maritimité? », in La maritimité.

Rosmorduc, J. et al.

1997 Histoire des sciences et techniques. CRDP Bretagne.

Vigarello, Georges

1988 Une histoire culturelle du sport. Paris : E.P.S., Laffont.

\section{ANNEXES}

\section{Glossaire}

Beachstart : Départ depuis la plage.

Footstraps : dispositifs pour attacher les pieds sur le flotteur.

Freeride : navigation libre, « just for fun ».

Freerace : hybridation entre freeride et course.

Freestyle : style libre.

Funboarder : utilisateur d'une planche à voile « fun », plutôt orienté plaisir, « éclate », vitesse, qu'ascétisme (compétition).

Gun : petit flotteur.

In and out : littéralement allers/retours à l'allure la plus simple et la plus rapide.

Indoors : compétitions en salle.

Jauge : c'est l'ensemble des facteurs limitant et/ou contraignant le développement des flotteurs autorisés à régater entre eux (longueur, poids, épaisseur). 
Maxis open : ce sont des bateaux de 18 mètres emmenés par un solitaire ou un équipage en course transatlantique ou autour du monde.

No nose : avant peu volumineux (littéralement « sans nez »).

Pan am : comparable aux longboard en surf.

Pin tail : arrière pointu.

Prototypes Open : flotteurs fabriqués à l'unité, dans la contrainte de la jauge du même nom, à des fins de régates.

Raceboard : long flotteur de compétition.

Shaper : désigne la personne qui donne une forme à partir d'un pain de mousse.

Scoop et outline : renvoient à la forme du flotteur (plat, banané, tendu, concave...).

Waterstart : départ dans l'eau.

Waveriders : chevaucheurs de vagues.

\section{NOTES}

1. C'est le cas du chantier Chabiland de Plouer sur Rance qui lancera l'Alusurf.

2. Les termes en italique sont définis dans le glossaire.

3. Nous pouvons relever que les catégories de poids sont alors nombreuses en planche à voile : au nombre de 3 en Open (les lourds regroupent les plus de $75 \mathrm{~kg}$ ), elles seront réduites à deux par la suite (plus ou moins de $70 \mathrm{~kg}$ ), puis elles disparaissent.

4. Ce qui semble, à la relecture, plus simple à mettre en pratique qu'à didactiser ou même formuler.

5. Les spectacles de P. Découflé portent par exemple cet imaginaire d'élévation et d'apesanteur.

6. Le terme est ici impropre et qualifie le groupe des planchistes confirmés opposé à celui des débutants moyens.

7. Les spécialistes me pardonneront d'occulter les indéniables succès populaires de la Torche ou les indoors de Marseille ou Bercy.

\section{RÉSUMÉS}

Entre le corps et les techniques se joue une dialectique complexe : l'homme produit le milieu qui l'entoure; la nature et la culture exercent à leur tour des contraintes sur les savoirs et savoirfaire ainsi que sur les représentations sociales. L'étude de la planche à voile et des véliplanchistes inscrit les corporéités techniques humaines -motricité et esthétique- comme produits de culture, en interactions permanentes

\section{Wind-surfing}

Body and techniques are linked by complex dialectics : Man creates its surrounding world; nature 
and culture, in turn, constrain knowledge and know-how as well as social representations. The study of wind-surfing inscribes human corporal techniques as products of culture for which muscular movement and aesthetics are in constant interaction

La tabla de windsurf.

Entre cuerpos y técnicas tiene lugar una dialéctica compleja: el hombre produce el medio que le rodea; la naturaleza y la cultura ejercen a su vez constricciones sobre los saberes y las destrezas, así como sobre las representaciones sociales. El estudio de la tabla de windsurf y de los surfistas inscribe las corporalidades técnicas humanas - motricidad y estética- en tanto que productos de la cultura, en interacciones permanentes.

INDEX

Mots-clés : corps, culture, contre-culture, socialisation, planche à voile

\section{AUTEUR}

\section{PHILIPPE LACOMBE}

Université de Bretagne Occidentale, Centre de recherche bretonne et celtique (CNRS) 29285 Brest Cedex 